\title{
Research on the Application of Computer Animation Technology in the Arrangement and Teaching of Rhythmic Gymnastics
}

\author{
Xiaoxuan Huang, Jun Zong \\ Jiangxi Science \& Technology Normal University, Jiangxi Province, Nanchang, 330013
}

Keywords: artistic gymnastics; arrangement and teaching; computer animation technology

\begin{abstract}
With the rapid development of computer technology and the increasing popularity of the network, more and more new teaching media are widely used in the field of education. For example, according to the teaching characteristics of rhythmic gymnastics, the use of computer-generated audio-visual media courseware has become a trend. The paper analyzes the difficulties in the arrangement and teaching of rhythmic gymnastics in detail, explores the application advantages and application conditions of computer animation technology in the arrangement and teaching of rhythmic gymnastics, aiming at promoting the development of rhythmic gymnastics education in China.
\end{abstract}

\section{Introduction}

In order to promote the development of China's sports industry and improve the physical fitness of the people, the State Council issued the "Several Opinions on Accelerating the Development of Sports Industry to Promote Sports Consumption" in 2014, which explicitly proposed to actively create a social atmosphere that values sports, supports sports, and participates in sports. The national fitness is promoted to the national strategy. Artistic gymnastics is an important sports project. Long-term practice can not only shape good body shape, but also enhance physique and promote cerebral development. However, due to the inherent attributes of rhythmic gymnastics, there are still many difficulties in the actual arrangement and teaching. The timely birth and introduction of computer animation technology provides an opportunity to solve these difficulties.

\section{The Concept of Computer Animation}

Animation is the use of human visual residual characteristics, a series of static pictures through a continuous play, visually creates a dynamic picture. With the maturity of computer technology, animation has undergone earth-shaking changes in both the concept of creation and the way of creation. The animations in people's mouths today mainly refer to computer animation. Computer animation refers to a series of dynamic images generated by computer graphics technology, using a combination of art, mathematics, physics and artificial intelligence, and programming or some related animation software. With the continuous expansion of animation in the field of application, animation has not only referred to animated movies and TV films with a certain plot in the traditional sense, but also refers to non-physical shooting used in commercial, industrial, educational and other fields. Screen works. Computer animation can be divided into two-dimensional animation and three-dimensional animation in the type. At present, three-dimensional animation is applied more in the arrangement and teaching of rhythmic gymnastics.

\section{The Difficulty in the Arrangement and Teaching of Rhythmic Gymnastics}

Rhythmic Gymnastics is a relatively new type of women's competitive sports. It originated in Europe in the late 19th and early 20th centuries and was introduced to China by the former Soviet Union 40 or 50 years later. Rhythmic gymnastics has a good effect on body shape and temperament training. It has been widely welcomed by the Chinese people, and many areas have carried out the 
teaching of rhythmic gymnastics. In the rhythmic gymnastics arrangement, it is often necessary to go through a relatively long process. The first is to carefully conceive, then the specific arrangement, and then the actual exercise. Finally, in order to achieve better results, it is often necessary to conduct one or several times on the basis of actual exercises. Undoubtedly, this will greatly cost teachers and students time and energy. In the teaching of rhythmic gymnastics, teachers are more likely to perform action demonstrations in front of students in the teaching process, so they cannot have a large amount of time to observe the students' learning situation, let alone find out that each student is insufficient in the learning process. At the same time, in the process of rhythmic gymnastics teaching, teachers may not be perfect in some actions, and even some action teachers with higher difficulty coefficients can't do it themselves. These conditions will greatly affect the teaching effect of rhythmic gymnastics.

\section{The Application Advantages of Computer Animation Technology in the Arrangement and Teaching of Rhythmic Gymnastics}

Because computer animation technology is used to simulate all the movements of rhythmic gymnastics, the process of rhythmic gymnastics is completed on the computer, which makes all scenes, lights, instruments, cameras and demonstrators through some related animation software. To achieve this, it can greatly save the time and energy of teachers and students and greatly save the school's expenditure on artistic gymnastics education.

Computer 3D animation uses some animation software to make mannequins in the calculation, and manipulates the human body model to simulate gymnastics movements to create corresponding animations. Since all of this is done on a computer, there is no problem that the three-dimensional animation of the computer does not have a standard motion of the demonstrator, and the technical level is not high enough, resulting in poor quality of the demonstration. Moreover, thanks to the three-dimensional technology, students can carefully observe the demonstrators from any angle in the three-dimensional space, so as to better improve their gymnastics movements. This advantage is difficult to achieve with many other aids. If you want to use video to achieve this requirement, you must use multiple cameras to perform multi-angle simultaneous shooting of the gymnastics demonstrators and the cameras can not interfere with each other, or use a camera to perform the gymnastics demonstrations. The difficulty of operation can be seen from multiple shootings. However, even if it is, it will not be able to achieve 360-degree shooting due to technical limitations. With 3D animation, it is only necessary to move the position of the analog camera on the computer to make it easy for the gymnastics demonstrations. You show the effect of the action at all angles.

Depending on the requirements of the teaching task and the learning situation of each student, the teacher can teach different ways by changing the speed of the computer animation. For simple rhythmic gymnastics movements, teachers can speed up the demonstration speed of computer animation to quickly demonstrate teaching; for more difficult rhythmic gymnastics movements, teachers can appropriately slow down the demonstration speed of computer animation for slow demonstration teaching. And teachers can also use manual demonstration, automatic demonstration and rapid demonstration, and pause the computer animation at any time according to the needs of the teaching situation. At the same time, because computer animation uses computer technology, it also has the technical advantages of high speed, high precision and high storage. This is also unmatched by other art gymnastics aids. For example, in terms of storage, if you use computer animation to simulate the movements of rhythmic gymnastics, you can use a small CD to demonstrate the whole rhythmic gymnastics movements, and even include some technical movements, which can be stored. . Moreover, since this storage method is stored in a digital manner, there is no deterioration in quality due to frequent use. As long as you want to learn gymnastics in the form of computer animation, you can take the disc out and carry it out at any time and anywhere. Learn.

In traditional rhythmic gymnastics teaching, teachers often stand in front of students as demonstrators of gymnastics movements. In the teaching process, the teacher spends more time on the demonstration of gymnastics and the explanation of gymnastics movements, so that there is not 
enough time to understand the students' learning situation, and they can not find out their shortcomings in time and take corresponding solutions. Upgrade them. The application of computer animation art to the arrangement and teaching of rhythmic gymnastics has greatly alleviated this embarrassing situation. To a certain extent, this is to withdraw the teaching from the teaching process, so that teachers can conduct a comprehensive survey of students from a bystander perspective. Once the students are found to be not perfect in which gymnastics movements are not perfect, the teacher can pause the computer animation, and then give them detailed explanations on those gymnastics movements, or repeatedly play back the movements on the computer animation, so that the students can follow the practice repeatedly.

\section{The Application Conditions of Computer Animation Technology in the Arrangement and Teaching of Rhythmic Gymnastics}

Since computer animation technology is used in the arrangement and teaching of rhythmic gymnastics, this requires the relevant teachers to have certain computer operation capabilities. With the development of computer technology and the widespread use of the Internet, people are becoming more and more comfortable with the use of computers. For this problem, as long as the relevant teachers learn some computer related knowledge and practice it, it can be easily solved.

At present, basically every school has been equipped with computers, and even some schools have specialized dance training rooms equipped with large-screen TVs or projectors, which makes it easier to apply computer animation technology to rhythmic gymnastics.

3D animation is an important part of computer animation, and it is widely used in the arrangement and teaching of rhythmic gymnastics. For ordinary teachers, making 3D animations is often difficult. At this time, it is necessary to seek the help of people who understand both artistic gymnastics and three-dimensional animation, and let them make three-dimensional animation of rhythmic gymnastics. Or the rhythmic gymnastics teacher can also find people who don't understand art gymnastics but understand three-dimensional animation, and the three-dimensional animation of the rhythmic gymnastics that he worked together.

\section{References}

[1] Xie Lin. Function and method: physical training in college rhythmic gymnastics teaching [J]. Chinese and foreign entrepreneurs, 2016 (33): 204-205.

[2] Wang Weixiao. Adolescent physical training method in artistic gymnastics teaching [J]. Movement, 2015(24):98+110.

[3] Wang Cong.The Teaching Method and Application of Rhythmic Gymnastics in Chinese Universities[J].Journal of Wuhan Institute of Physical Education,2013,47(01):79-81.

[4] Pu Ming, Jia Shangmin, Liu Wei. Application of Computer 3D Animation Technology in Vaulting Teaching [J]. Journal of PLA Institute of Physical Education, 2000(02): 89-90. 